\title{
CO-EVOLUTIONARY LEARNING IN STRATEGIC ENVIRONMENTS
}

\author{
Akira Namatame, Naoto Sato, Yukikazu Murakami \\ Dept. of Computer Science \\ National Defense Academy, Yokosuka, JAPAN \\ E-mail:nama@nda.ac.jp
}

\begin{abstract}
An interesting problem is under what circumstances will a collection of interacting agents realize efficient collective actions. This question will depend crucially on how self-interested agents interact and how they learn from each other. We model strategic interactions as dilemma games, coordination games or hawk-dove games. It is well known that the replicator dynamics based on natural selection converge to an inefficient equilibrium. In this paper, we focus on the effect of coevolutionary learning. Each agent is modeled to learn interaction rules defined as the function of own strategy and the strategy of the neighbor. We show that a collection of interacting agents converges into equilibrium in which the conditions of efficiency and equity are satisfied. We investigate interaction rules acquired by all agents and show that they share several rules with the common features to sustain equitable social efficiency. This paper also presents a comparative study of two evolving populations, one in a spatial environment, and the other in a small-world environment. The effect of the environment on the emergence of social efficiency is studied. The small-world environment is shown to encourage the emergence of social efficiency further than the spatial structure.
\end{abstract}

Keywords: Co-evolution, dilemma game, coordination game, hawkdove game, anti-coordination game, local interaction, small-world network, efficiency, fairness 


\section{Introduction}

In many applications it is of interest to know which strategies can survive in the long run. While the concept and techniques of game theory have been used extensively in many diverse contexts, they have been unsuccessful in explaining how agents realize if a game has many equilibria $^{8}$. Introspective or educative theories that attempt to explain equilibrium selection problem directly at the individual decision-making level impose very strong informational assumptions. The game theory is also not able to address issues on how agents know which equilibrium should be realized when games have multiple equally plausible equilibria $^{3}$. The game theory is also not able to provide answer in explaining how agents should behave in order to overcome an inefficient equilibrium situation ${ }^{7}$.

One of the variations involves the finitely iterated games. The standard interpretation of game theory is that the game is played exactly once between fully rational individuals who know all details of the game, including each other's preferences over outcomes. Evolutionary game theory, instead, assumes that the game is repeated many times by individuals who are randomly drawn from large populations ${ }^{15,18}$. An evolutionary selection process operates over time on the population distribution of behaviors. It is also of interest to know which strategies can survive in the long run. According to the fundamental theorem principle of natural selection, more fitter behavior is selected.

The evolutionary dynamic model with the assumption of uniform matching can be analyzed using replicator dynamics ${ }^{4}$. The criterion of evolutionary equilibrium highlights the role of mutations. The replicator dynamics, highlight the role of selection. Evolutionary game theory assumes that the game is repeated by individuals who are randomly drawn from large populations ${ }^{6}$. However, the growing literatures on evolutionary models have not considered learning at individual levels ${ }^{9}$. They treat agents as automata, merely responding to changing environments without deliberating about individuals' decisions. Within the scope of our model, we treat models in which agents make deliberate decisions by applying rational reasoning about what to do and also how to decide ${ }^{13,14}$. Two features of this approach distinguish it from the introspective approach. First, agents are not assumed to be so rational or knowledgeable as to correctly guess or anticipate the other agent's 
strategies. Second, an explicit dynamic process is specified describing how agents adapt their strategies as they repeat the games.

An interesting problem is under what circumstances agents with individual learning may converge to some particular equilibrium ${ }^{1,2}$. We endow our agents with some simple way of learning and describe the evolutionary dynamics that magnifies tendencies toward better situation. By incorporating a consideration of how agents interact into models we not only make them more realistic but we also enrich the types of aggregate behavior that can emerge $\mathrm{e}^{10,11,12}$. It is an important question to answer the following question: how the society groups its way towards an efficient equilibrium in an imperfect world when self-interested agents learn from each other.

The term evolutionary dynamics often refers to systems that exhibit a time evolution in which the character of the dynamics may change due to internal mechanisms. In this paper, we focus on evolutionary dynamics that may change in time according to certain local rules. Evolutionary models can be characterized both by the level at which the mechanisms are working and the dimensionality of the system. We use the evolutionary models based on microscopic individuals who interact locally ${ }^{16}$. The search for evolutionary foundations of game-theoretic solution concepts leads from the notion of an evolutionarily stable strategy to alternative notions of evolutionary stability to dynamic models of evolutionary processes. The commonly used technique of modeling the evolutionary process as a system of a deterministic difference or differential equations may tell us little about equilibrium concepts other than that strict Nash equilibrium are good. We can attempt to probe deeper into these issues by modeling the choices made by agents with their learning models.

We focus on collaborative learning in strategic environments, Noncooperative games are classified into dilemma games coordination games, and hawk-dove games. It is well known that natural selection does leads to inefficient equilibria in these games. In this paper each agent learns interaction rules by repeating games. We provide a general class of adaptation models and relate their asymptotic behavior to equilibrium concepts. We assume agents behave myopically, and they evolve their interaction rule over generation. They learn from the most successful strategy of their neighbor. Hence their success depends in large part on how well they do in their interactions with their neighbors. If the neighbor is doing well, the rule of the neighbor can be imitated, and in 
this way successful rule can spread throughout a population, from neighbor to neighbor.

We consider two fundamental models of interaction, local interaction with the lattice model and small-world model ${ }^{17}$. We show that all agents mutually learn acquire the common-rule, which lead to social efficiency. We also investigate acquisition by rules, and show that those rules of agents are categorized into a few rules with some commonality.

\section{Interaction with Lattice Model and Small-World Networks}

It is important to consider with whom an agent interacts and how each agent decides his action depending on others' actions. In order to describe the interactions among agents, we may have two fundamental models, random matching and local matching. The approach of random (or uniform) matching is modeled as follows: In each time period, every agent is assumed to match (interact) with one agent drawn at random from a population.

An important assumption of the random matching is that they receive knowledge of the current strategy distribution. Each agent makes his rational decision strategy based on a sample of information about what other agents have done in the previous time period. Agents are able to calculate best replies and learn the strategy distribution of play in society. Agents may adapt based on the aggregate information representing the current status of the whole system (global adaptation). In this case, each agent chooses an optimal decision based on aggregate information about how all other agents behaved in the past. An agent calculates her reward and plays her best response strategy. An important assumption of global adaptation is that they receive knowledge of the aggregate.

In many situations, however, agents are not knowledgeable so as to correctly guess or anticipate other agents' actions, or they are less sophisticated and that they do not know how to calculate best replies ${ }^{8}$. We assume that a spatial environment is a more realistic representation since interactions in real life rarely happen on such a macro scale; Spatial interaction is generally achieved through the use of a 2D grid as shown in Figure 1(a), with each agent inhabiting a cell on the grid. Interaction between agents is restricted to neighboring cells. This may allow for individuals, which may have been eliminated if assessed against all players, to survive in a niche. 
The recognition of the importance of spatial interactions has led to many exploring and extending aspects of it. Nowak and May focused upon evolutionary niching and the pattern of emergence of cooperation in the spatial environment ${ }^{13}$. With local adaptation each agent is modeled to adapt to his neighbors. The hypothesis of local adaptation also reflects limited ability of agents' parts to receive, decide, and act based upon information they receive in the course of interaction. Agents observe the current performance of their neighbors, and learn from the most successful agent. Agents are less sophisticated in that they do not know how to calculate best replies and are using other agent's successful strategies as guides for their own choices. Each agent interacts with the agents on all eight adjacent squares and imitates the strategy of any better performing one. In each generation, each agent attains a success score measured by its average performance with its eight neighbors. Then if an agent has one or more neighbors who are more successful, the agent converts to the rule of the most successful neighbor.

Complex networks describe a wide range of systems in nature and technology. They can be modeled as a network of nodes where the interactions between nodes are represented as edges. Recent advances in understanding these networks revealed that many of the systems show a small-world structure. Watts and Storogatz introduced a small-world network which transforms from a nearest neighbor coupled system to a random coupled network by rewiring the links between the nodes ${ }^{17}$. Two parameters are used to describe the transition. The mean path length L, which specifies the global property of the network, is given as the mean of the shortest path between all pairs of vertices. In contrast, the clustering coefficient $C$ characterizes the local property of the system and can be calculated as the fraction of the connections between the neighbors of a node divided by the number of edges of a globally coupled neighborhood, averaged over all vertices.

Consider one lattice model in which each node is coupled with its nearest neighbors as shown in Figure 1(b). It has a large mean path length and a high clustering coefficient. If one rewires the links between the node with a small probability the local structure of the network remains almost conserved keeping the clustering coefficient contrast. In contrast, due to the introduction of short cuts by the rewiring procedure the mean path length becomes strongly reduced. Networks with these properties are small-world networks. Further increase of the rewiring probability results in a random coupled network with a short mean path 
length and a low clustering coefficient.

Figure 1. The topology of interaction

(a) Local interaction with a lattice model

(b) Interaction with a small-world network (Illustration of one-lattice model)
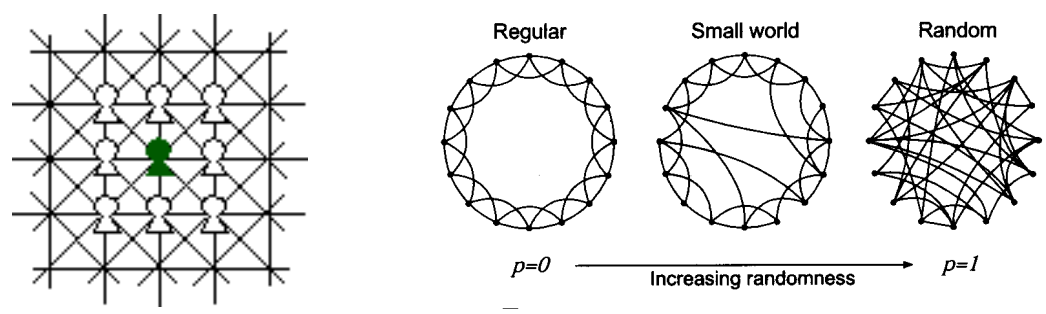

\section{Learning Models}

Game theory is typically based upon the assumption of a rational choice ${ }^{8}$. In our view, the reason for the dominance of the rational-choice approach is not that scholars think it to be realistic. Nor is game theory used solely because it offers good advice to a decision maker, because its unrealistic assumptions undermine much of its value as a basis for advice. The real advantage of the rational-choice assumption is that it often allows deduction.

The main alternative to the assumption of rational choice is some form of adaptive behavior. The adaptation may be at the individual level through learning, or it may be at the population level through differential survival and reproduction of the more successful individuals. Either way, the consequences of adaptive processes are often very hard to deduce when there are many interacting agents following rules that have nonlinear effects. Among many adaptive mechanisms that have been discussed in the literature on learning are classified as follows:

\section{(1) Best response learning}

In most game theoretic models, agents have perfect knowledge of the consequences of their decision. An important assumption of bestresponse learning is that they receive knowledge of the current strategy distribution. Agents can calculate their best strategy based on information about what other agents have done in the past. Then agents 
gradually learn the strategy distribution in the society. Agents adopt actions that optimize their expected payoff given what they expect others to do. In this learning model, agents choose the best replies to the empirical frequencies distribution of the previous actions of the others.

\section{(2) Reinforcement learning}

Agents tend to adopt actions that yielded a higher payoff in the past, and to avoid actions that yielded a low payoff. Payoff describes choice behavior, but it is one's own past payoffs that matter, not the payoffs of the others. The basic premise is that the probability of taking an action in the present increases with the payoff that resulted from taking that action in the past.

\section{(3) Evolutionary learning}

Agents with higher payoff are at a productive advantage compared to agents who use low-payoff strategies, hence the latter decrease in frequency in the population over time (natural selection). In the standard model of this situation agents are viewed as being genetically coded with a strategy and selection pressure favors agents that are fitter, i.e., whose strategy yields a higher payoff against the population. The idea of using a genetic algorithm (GA) to create strategies has been developed further by Lindgren ${ }^{9}$. He showed that strategies could be made more robust by seeding the initial population with expert, hand-coded strategies.

\section{(4) Social learning}

Agents learn from each other with social learning. For instance, agents may copy the behavior of others, especially behavior that is popular to yield high payoffs (imitation). In contrast to natural selection, the payoffs describe how agents make choices, and agents' payoff must be observable by others for the model to make sense. The crossover is a kind of social learning.

\section{Evolutionary Dynamics with Individual Learning}

We make a distinction between evolutionary systems and adaptive systems. The equations of motion in an evolutionary system reflect the basic mechanisms of biological evolution, i.e., inheritance, mutation, and selection. In an adaptive system, other mechanisms are allowed as well, 
e.g., modifications of strategies based on individual forecasts on the future state of the system. But, increasing the possibilities for individualistic rational behavior does not necessarily improve the outcome for the species to which the individual belongs in the long run.

The introduction of spatial dimensions, so that individuals only interact with those in their neighborhood, may affect the dynamics of the system in various ways. The possibility of space-temporal structures may allow for global stability where the mean-field model (random matching) would be unstable. The presence of these various forms of spacetemporal phenomena may, therefore, also alter the evolutionary path compared with the mean-field model and we may see other strategies evolve.

Different aspects of the evolutionary behavior have been investigated by many researchers: (i) by varying the payoff matrix of the game, (ii) by introducing spatial dimensions, and (iii) by introducing co-evolution. An important aspect of evolution is the learning strategy adapted by individuals ${ }^{3}$. Evolution in the hawk-dove game, for instance, drives the population to an equilibrium polymorphism state. But this symmetrical mixed equilibrium of hawk-dove is so inefficient that it is far from optimal.

The term evolutionary dynamics often refers to systems that exhibit a time evolution in which the character of the dynamics may change due to internal mechanisms. In this paper, we focus on evolutionary dynamics that may change in time according to certain local rules of individuals. Evolutionary models can be characterized both by the level at which the mechanisms are working and the dimensionality of the system. Therefore we describe the evolutionary dynamics specifying microscopic behavior with individuals learning.

The search for evolutionary foundations of game-theoretic solution concepts leads from the notion of an evolutionarily stable strategy to alternative notions of evolutionary stability to dynamic models of evolutionary processes. The commonly used technique of modeling the evolutionary process as a system of a deterministic difference or differential equations may tell us little about equilibrium concepts other than that strict Nash equilibrium is good. We can attempt to probe deeper into these issues by modeling the choices made by the agents with their own internal models. We also focus on dynamical systems described by equations of motion that may change in time according to certain rules, which can be interpreted as crossover operations. Each agent learns to 
acquire the rule of interaction in the long-run. Non-cooperation games can be categorized into, dilemma games coordination games, HawkDove games and minority games. It is known that natural selection does not lead to social efficiency in these games. We show that all agents mutually learn to cooperate which result in social efficiency.

\section{Learning Coupling Rules}

In most game theoretic models, agents calculate their best strategy based on information about what other agents have done in the past. Then agents may gradually learn the equilibrium strategy. A number of evolutionary models based on the iterated general non-cooperation games have been proposed. Many dynamical systems and evolutionary models have been constructed with the $\mathrm{PD}^{1}$. Yao applied a genetic algorithm (GA) to the iterated Prisoner's Dilemma and used a bit-string representation of finite memory strategies ${ }^{21}$.

We use the different approach. In the models that we discuss here, the equations of motion for the different individuals are usually coupled, which means that we have co-evolutionary systems. The success or failure for a certain type of individual depends on which other individuals are present. In this case, there is not a fixed fitness landscape in which the co-evolutionary dynamics climbs toward increasing to fitness. This ever-changing character of the world determining the evolutionary path allows for evolutionary phenomena.

Co-evolutionary dynamics differ, in this sense, from the common use of the genetic algorithm, in which a fixed goal is used in the fitness function and where there is no coupling between individuals. In the genetic algorithm, the focus is on the final result what is the best or a good solution. In models of co-evolutionary systems, one is usually interested in the transient phenomenon of evolution.

Each strategy in the repeated game is represented as a binary string so that the genetic operators can be applied. In order to accomplish this we treat each strategy as deterministic bit strings. We use a memory of one or two, which means that the outcomes of the previous one or two moves are used to make the current choice. We assume that $0=\mathrm{S}_{1}$ and $1=\mathrm{S}_{2}$ then as Figure 2(a) shows, there are four possible outcomes between two agents for each move $\mathrm{S}_{1} \mathrm{~S}_{1}(0,0), \mathrm{S}_{1} \mathrm{~S}_{2}(0,1), \mathrm{S}_{2} \mathrm{~S}_{1}(1,0), \mathrm{S}_{2} \mathrm{~S}_{2}(1,1)$. We can fully describe a deterministic strategy by recording what the strategy will do in each 
of the 4 different situations that can arise in the iterated game. Since no memory exists at the start, an extra 2 for 4 bits are needed to specify a hypothetical history. Each rule can be defined by a 6 bit string as shown in Figure 2(b).

At each generation, agents repeatedly play the game for $T$ iterations. Agent $i, i \in[1 \ldots \mathrm{N}]$ uses a binary string $i$ to choose his strategy at iteration $t, t \in .[1 \ldots \mathrm{T}]$. Each position of a binary string in Figure 2(b) as follows: The first position, $p_{1}$ encodes the action that agent takes at iteration $t=1$. A position $p_{j}, j \in[2,3]$ encodes the memories that agent $i$ takes at iteration $t-1$ and his opponent. A position $p_{j}, \mathrm{j} \in[4 \ldots 7]$, encodes the action that agent $i$ takes at iteration $t>1$, corresponding to the position $p_{j}, j \in[2,3]$. An agent $i$ compares the position $p_{j}, j \in[2,3]$, decides the next action.

Figure 2. An interaction rule of memory one

(a) Coupling Rule

\begin{tabular}{|c|c|c|c|}
\hline \multirow{2}{*}{ bit } & \multicolumn{2}{|c|}{ previous strategy } & \multirow{2}{*}{ next strategy } \\
\cline { 2 - 3 } & Own & Opp & \\
\hline 4 & 0 & 0 & $\#$ \\
\hline 5 & 0 & 1 & $\#$ \\
\hline 6 & 1 & 0 & $\#$ \\
\hline 7 & 1 & 1 & $\#$ \\
\hline
\end{tabular}

(b) Rule Representation

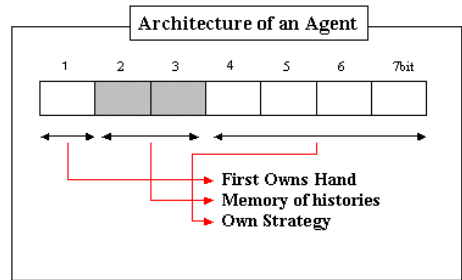

Each agent mimics the rule of the most successful neighbor. We arrange agents for an area of $20 \times 20(\mathrm{~N}=400$ agents) with the lattice model as shown in Figure 1(a) with no gap, and four corners and end of an area connect it with an opposite side. At each time period $t$, each agent plays with his 8 neighbors. At the next time period, each agent mimic the interaction rule of the most successful neighbor who obtain the highest payoff.

\section{Simulation Results}

Non-cooperation games can be categorized into dilemma games coordination games, hawk-dove games. It is known that equilibrium situations led by natural selection is far from social efficiency. A genetic algorithm is used to evolve strategies. A generation involves each player playing with 8 neighbors with the spatial model or some proportion of partners to interact are chosen from all other members in the population 
with the model of small-world networks. The iterated game is played fifty times between each agent. The fitness of an agent is the average payoff it achieved over repeating games. Mutation of random alleles may occur with a probability of 0.01 for all cases.

\section{(1) Dilemma Game}

Many works on evolution of cooperation have been focused on dilemma games, which is formulated as follows: Each agent faces the problem of selecting one of two decisions, cooperate $\left(\mathrm{S}_{1}\right)$ or defect $\left(\mathrm{S}_{2}\right)$. The payoff for each decision depends on the decisions of the other agent. Table 1 shows the payoffs for all the possible combinations of decisions. The most startling effect of the iterated Prisoner's Dilemma simulation, as observed by Axelrod ${ }^{1}$, is the fact that a group of purely egotistical individuals, working towards nothing but improving themselves can lead to a population which is actually highly cooperative.

Each pair of agents interacts 50 times at one generation. Figure 3(a) shows the ratio of agents who chose the cooperative strategy $S_{1}$ with the lattice model. After few generations the ratio of the cooperative becomes to be 0.85 . Initially the ratio of defective strategy increases, however, it is quickly wiped out and more cooperative opponents obtain higher payoffs, and the population exhibits reciprocal cooperation. Figure 3(b) shows the same experiment in a small-world network model. There are a couple of important differences in this graph and the graph obtained using the spatial environment (Figure 3(a)). Figure 3(b) shows that it is actually easier for cooperation to evolve in a small-world network environment. As a result the cooperative strategy could be realized with the dilemma game after a few generation. The cooperation is clearly more stable in the small-network environment.

At beginning, each agent has a different interaction rule specified by the 4 bits information. All rules learnt by 400 agents, which were aggregated into only one type as shown in Table 3.

Table 1 . The payoff matrix of a dilemma game

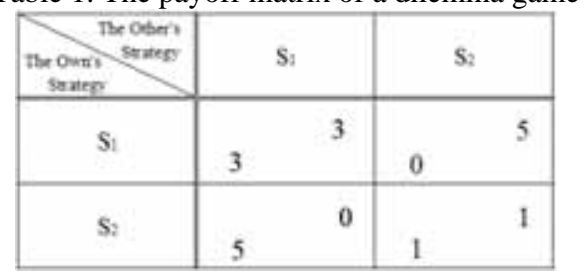


Figure 3. The ratio of cooperation in iterated dilemma games

(a) Local interaction with a lattice model (b) Interaction with a small-world network
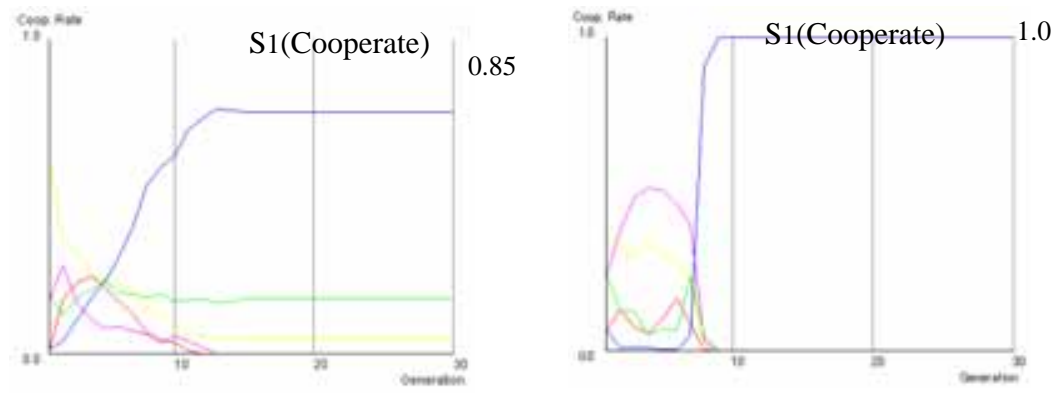

Table 2. Learnt Rules by 400 agents in small-world environment

\begin{tabular}{|c|c|c|c|c|c|}
\hline \multirow{2}{*}{$\begin{array}{c}\text { Initial } \\
\text { strategy }\end{array}$} & \multicolumn{4}{|c|}{ Array Location } & \multirow{2}{*}{$\begin{array}{c}\text { Number of } \\
\text { Agents }\end{array}$} \\
\hline & 4 & 5 & 6 & 7 & \\
\hline 0 & 0 & 1 & 1 & 1 & 400 \\
\hline
\end{tabular}

Table 3. Learnt Interaction rule

\begin{tabular}{|c|c|c|c|}
\hline \multirow{2}{*}{ bit } & \multicolumn{2}{|c|}{ previous strategy } & \multirow{2}{*}{ strategy at t } \\
\cline { 2 - 3 } & Own & Opp & \\
\hline 4 & 0 & 0 & 0 \\
\hline 5 & 0 & 1 & 1 \\
\hline 6 & 1 & 0 & 1 \\
\hline 7 & 1 & 1 & 1 \\
\hline
\end{tabular}

Figure 4. The state transitions specified by the rule in Table 3.

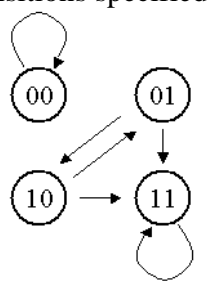

The acquired rule specified as " 0111 " in Table 3 can be interpreted as follows: If agents behave as "cooperate", then both agents behave "cooperate", however one of them "defect", then both agents behave "defect". The state transition of this learnt rule is illustrated in Figure 4 as the state transition diagram. There are two absorption points " 00 " and "11". Since each agent also acquires the rule to behave "cooperate" at the first play of each generation as shown in Table 2, they remain at the 
absorption points "00".

\section{(2) Coordination Games}

The coordination game with the payoff matrix in Table 4 has two equilibria with the pairs of the pure strategies $\left(S_{1}, S_{1}\right),\left(S_{2}, S_{2}\right)$, and one equilibrium of the mixed strategy. The most preferable equilibrium, Pareto-dominance is ( $S_{1}, S_{1}$ ), which dominates the other equilibrium. There is another equilibrium concept, the risk-dominance, and $\left(S_{2}, S_{2}\right)$ risk-dominates $\left(S_{1}, S_{1}\right)$. How do agents choose their strategy when the equilibria of Pareto-dominance and the risk-dominance are different? With such indigenous selection of strategy, the question is whether the society of agents may select the socially efficient Pareto-optimal strategy.

Figure 5(a) shows the ratio of agents to choose the Pareto-optimal strategy $S_{1}$ with the lattice model. After few generations the ratio of the Pareto-optimal strategy becomes to be 0.85. Figure 5(b) shows the same experiment using a small-world network framework. There are a couple of important differences in this graph and the graph obtained using the spatial environment (Figure 5(a)). Figure 5(b) shows that it is easier for the Pareto-optimal strategy to evolve in a small-world network environment. As a result Pareto optimal strategy could be spread out after a few generation.

At beginning, each agent has a different interaction rule specified by the 4 bits information. In Table 5, we show the rules learnt by 400 agents, which are aggregated into only one type. After 10 generations, all rules of agents were converged into one rule as shown in Table 5 . The acquired rule specified as " 0111 " in Table 5 can be interpreted as follows: If both agents choose the Pareto-optimal strategy, then they choose the same one, however if one of them chooses the risk-dominant strategy, then both agents choose the risk-dominant strategy. The state transition of the learnt rule is illustrated in Figure 6 as the state transition diagram. There are two absorption points "00" and "11". Since each agent also acquires the rule to choose "Pareto-optimal strategy" at the first play of each generation as shown in Figure 6, they remain at the absorption points "00". 
Table 4. Payoff matrix of a coordination game

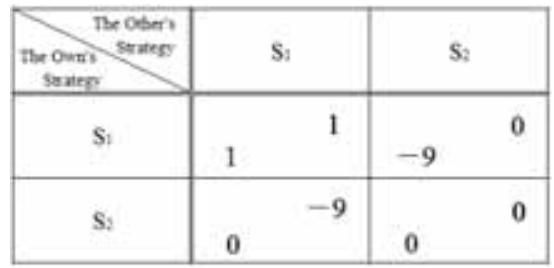

Figure 5. The ratio of Pareto-optimal strategy (S1) in iterated coordination games (a) Local interaction with a lattice model (b) Interaction with a small-world network

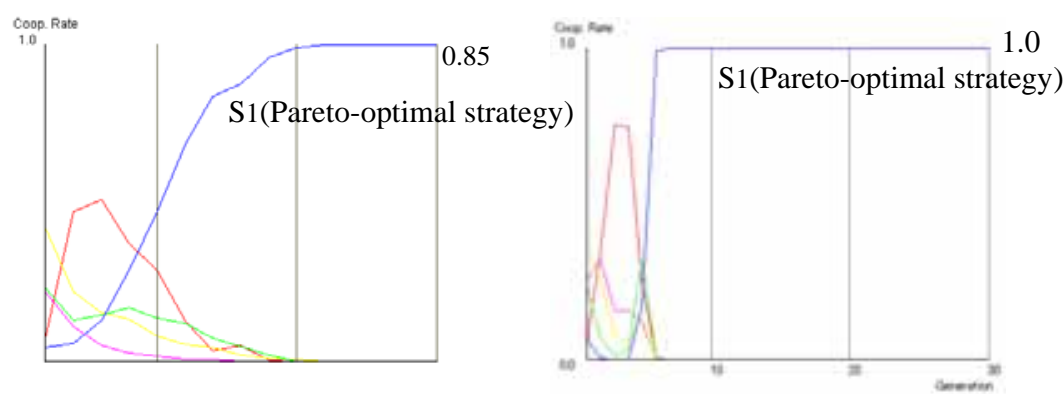

Table 5. Learnt Rules by 400 agents in Interaction with a small-world network

\begin{tabular}{|c|c|c|c|c|c|}
\hline \multirow{2}{*}{$\begin{array}{c}\text { Initial } \\
\text { strategy }\end{array}$} & \multicolumn{4}{|c|}{ Array Location } & \multirow{2}{*}{$\begin{array}{c}\text { Number of } \\
\text { Agents }\end{array}$} \\
\hline & 4 & 5 & 6 & 7 & \\
\hline 0 & 0 & 1 & 1 & 1 & 400 \\
\hline
\end{tabular}

Table 6. Interaction rule

\begin{tabular}{|c|c|c|c|}
\hline \multirow{2}{*}{ bit } & \multicolumn{2}{|c|}{ previous strategy } & \multirow{2}{*}{ strategy at t } \\
\cline { 2 - 4 } & Own & Opp & \\
\hline 4 & 0 & 0 & 0 \\
\hline 5 & 0 & 1 & 1 \\
\hline 6 & 1 & 0 & 1 \\
\hline 7 & 1 & 1 & 1 \\
\hline
\end{tabular}

Figure 6. The state transitions specified by rules in Table 6 .

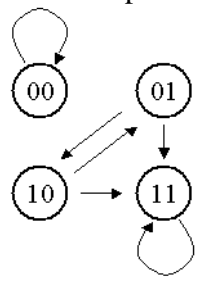




\section{(3) Hawk-Dove Game}

The hawk-dove game is formulated with the payoff matrix in Table 7. In this game, we suppose there are two possible behavioral types; one escalates the conflict until injury or sticks to display and retreats if the opponent escalates. These two types of behavior are described as "hawk" and "dove". There is the unique symmetric Nash equilibrium in mixed strategies, both agents use the strategy $S_{l}$ ('hawk') with probability $p=V / C$ and the strategy $S 2$ ('dove') with the probability $1-p=1-(V / C)$ [2]. Therefore if the cost of injury $\mathrm{C}$ is very large, the hawk frequency $(V / C)$ will be small. At equilibrium of the mixed strategy, the expected fitness is given at the level of $(V / 2)\{1-(V / C)\}$. If each agent chooses the strategy $S 2$ ('dove'), (however, the situation that both behave as doves are not equilibrium) he receives $V / 2$. This implies that the mixed-strategy results in inefficient equilibrium. And evolutionary game can realize Pareto optimal equilibrium but it has the possibility that an inferior equilibrium is chosen.

Figure 7(a) shows the ratio of agents to choose the strategy of Dove $\left(\mathrm{S}_{2}\right)$ with the lattice model. We set to $V=10, C=12$ in Table 7 . After few generations the ratio of the strategy of Dove becomes 0.95 . Figure 7(b) shows the same experiment, this time using a smallworld network framework.

In Table 8, we show the rules learnt by 400 agents, which are aggregated into only one type. After 10 generation, all rules of agents were converged into one rule as shown in Table 8 . The acquired rule specified as " 0001 " in Table 8 can be interpreted as follows: If both agents choose the Dove strategy, then they choose the same one, however one of them chooses the Hawk strategy, then both agents choose the Hawk strategy. The state transition of the learnt rule is illustrated in Figure 8 as the state transition diagram. There are two absorption points "00" and "11". Since each agent also acquires the rule to choose " the Dove strategy" at the first play of each generation as shown in Table 8, they remain at the absorption points "11". 
Table 7. The payoff matrix of the Hawk-dove game

\begin{tabular}{|c|c|c|}
\hline Ownit She Othe's & $\begin{array}{c}\text { S: } \\
(\mathrm{Hank})\end{array}$ & $\begin{array}{c}\text { S; } \\
\text { (Dove) }\end{array}$ \\
\hline $\begin{array}{c}\mathbf{S}_{1} \\
\text { (Hawk) }\end{array}$ & $\mathrm{N}-\mathrm{Cr}^{\mathrm{V} \cdot \mathrm{Cy} 2}$ & $v$ \\
\hline $\begin{array}{c}\text { S: } \\
\text { (Dove) }\end{array}$ & $\mathrm{v}$ & $\mathrm{v} / 2$ \\
\hline
\end{tabular}

Figure 7. The ratio of Dove (S2) in iterated dilemma games

$\begin{array}{lll}\text { (a) Local interaction with a lattice model } & \text { (b) Interaction with a small-world network }\end{array}$
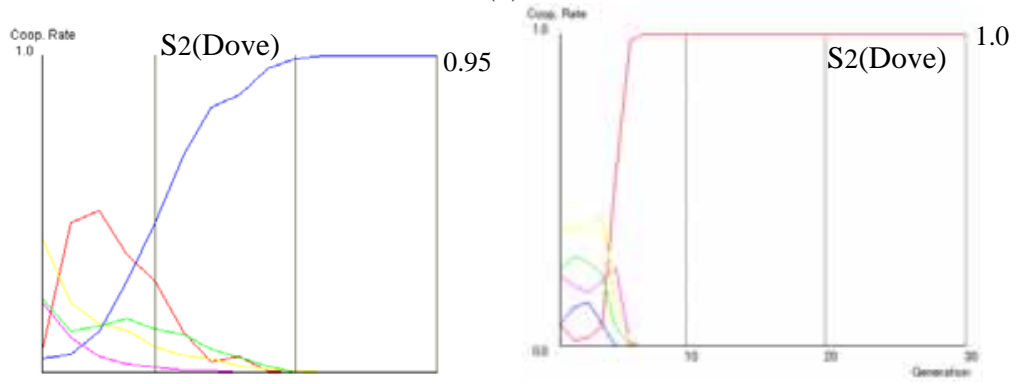

Table 8. Learnt Rules by 400 agents with a small-world network

\begin{tabular}{|c|c|c|c|c|c|}
\hline \multirow{2}{*}{$\begin{array}{c}\text { Initial } \\
\text { strategy }\end{array}$} & \multicolumn{4}{|c|}{ Array Location } & Number of \\
\cline { 2 - 6 } & 4 & 5 & 6 & 7 & Agents \\
\hline \hline 1 & 0 & 0 & 0 & 1 & 400 \\
\hline
\end{tabular}

Table 9. Interaction rule

\begin{tabular}{|c|c|c|c|}
\hline \multirow{2}{*}{ bit } & \multicolumn{2}{|c|}{ previous strategy } & \multirow{2}{*}{ strategy at t } \\
\cline { 2 - 3 } & Own & Opp & \\
\hline 4 & 0 & 0 & 0 \\
\hline 5 & 0 & 1 & 0 \\
\hline 6 & 1 & 0 & 0 \\
\hline 7 & 1 & 1 & 1 \\
\hline
\end{tabular}

Figure 8. The state transitions specified by rules in Table 8

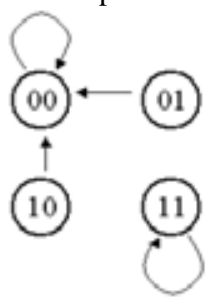




\section{Conclusion}

We focused on co-evolutionary dynamical systems described by equations of motion that may change in time according to rules. In the models that we discuss here, the equations of motion for the different individuals are usually coupled, which means that we have coevolutionary systems. The success or failure for a certain type of individual depends on which other individuals are present. In this case, there is not a fixed fitness landscape in which the evolutionary dynamics climbs toward increasing elevation, but a position that at one time is a peak may turn into a valley. This ever-changing character of the world determining the evolutionary path allows for complex dynamic phenomena. Co-evolutionary dynamics differ, in this sense, from the common use of the genetic algorithm, in which a fixed goal is used in the fitness function and where there is no interaction between individuals. In the genetic algorithm, the focus is on the final result what is the best or a good solution. In models of co-evolutionary systems, we consider the case of open-ended evolution.

We discussed the role of individual learning in realizing social efficiency. The hypotheses we employed here reflect the limited ability of interaction with the individual learning capability. The learning strategy employed here is a kind of meta-learning. One of the variations involves the finitely iterated game that has Nash equilibria of inferior strategies. It is illustrated that when the interaction architecture of the small-world network is added, evolution usually avoids this inferior state.

Our comparison of individuals playing the social games evolved in a spatial and small-world network environment has yielded some interesting results. It has been demonstrated in this paper that interaction on a small-world network framework encourages and promotes efficiency to a greater extent, and in a more stable way, than when interaction is performed on a spatial model. This suggests that efficiency will be easier to attain when exchanges are restricted to those in an open society rather than a closed society.

\section{References}

1. Axelrod, R., “The Complexity of Cooperation”, Princeton Univ. Press, 1997.

2. Arthur, W. B., "Inductive reasoning and Bounded Rationality", American Economic Review, vol.84, pp406-411, 1994. 
3. Challet, D, and Zhang. C., "Emergence of Cooperation and Organization in an Evolutionary Game”, Physica A246, 1997.

4. Fudenberg, D., and Levine, D., "The Theory of Learning in Games”, The MIT Pres, 1998.

5. Hammerstein, P. and Selten, R., "Game Theory and Evolutionary Biology", Handbook of Game Theory with Economic Applications, Vol.2, Auman, R., Hart, S (Eds), Elsevier Science, pp.931-962, 1994.

6. Hansaryi, J. and Selten, R., “A Game Theory of Equibrium Selection in Games”, MIT Press, 1988.

7. Hofbauer, J. and Sigmund, K., "Ecolutionary Games and Population Dynamics”, Cambridge Univ. Press, 1998.

8. Kandori, M. and Mailath, G., "Learning, Mutation and Long Run Equilibria in Games", Econometrica Vol.61, pp.29-56, 1993.

9. Kaniovski, Y., Kryazhimskii, A., and Young, H. “Adaptive Dynamics in Games Played by Heterogeneous Populations”, Games and Economics Behavior, 31, 50-96, 2000.

10. Lindgren, K., “Evolutionary Dynamics in Game-Theoretic Models”, The Economy as an Evolving Complex System II, pp.337-367, 1997.

11. Murakami, Y., Sato, H., and Namatame, A., "Co-evolution in Negotiation Games International Conference on Computational Intelligence and Multimedia Applications” pp241-245, 2001.

12. Murakami, Y., Sato, H., and Namatame, A., "Co-evolution in Hawk-Dove Games in a Mobile Environment” The $5^{\text {th }}$ Australia-Japan Joint Workshop on Intelligent \& Evolutionary Systems, Otago, New Zealand, pp 19-25, 2001.

13. Nowak, M. A., etc., "The Arithmetics of Mutual Help", Scientific American, June, 1995.

14. Nowak, M. A., and May, R. M., "Evolutionary Games and spatial Chaos”, Nature, 359, pp. 826-829, 1992.

15. Sipper, M., "Evolution of Parallel Cellular Machines”, Springer, 1996.

16. Smith, J. M., "Evolution and the Theory of Games”, Cambridge University Press, 1982.

17. Uno, K., and Namatame, A., "Evolutionary Behaviors Emerged through Strategic Interactions in the Large", GECCO'99 Workshop on Artificial life, 1999.

18. Watts, D. J., "Small Worlds”, Princeton University Press, 1999.

19. Weibull, J., “Evolutionary Game Theory”, The MIT press, 1996.

20. Yao, X., and Darwen, P., "The experimental study of N-player iterated prisoner's dilemma", Informatica, Vol.18:pp.435-450, 1994. 